\title{
5. IV-Revision: Welche Rolle spielen die Ärzte?
}

Inès Rajower

Korrespondenz:

Dr. med. Inès Rajower

Bundesamt für Sozialversicherungen

Effingerstrasse 20

CH-3003 Bern

Tel. 0313229047

Fax 0313223715

ines.rajower@bsv.admin.ch
Die Zahl der IV-Rentner/innen hat in den letzten zehn Jahren sehr stark zugenommen. Während 1996208000 IV-Renten ausbezahlt wurden, so waren es 10 Jahre später bereits 299000 . Im selben Zeitraum ist der Anteil der IV-Rentner/innen an der aktiven Bevölkerung von knapp 3,8 auf über 5,4 Prozent angestiegen, d.h., heute ist bereits jede zwanzigste Person IV-Rentenbezüger/in [1]. Renten, die wegen psychischer Erkrankung ausgerichtet werden, haben dabei überdurchschnittlich stark zugenommen. Heute sind rund 40 Prozent der Neurenten auf psychische Erkrankungen zurückzuführen (Abb. 1). Diese Entwicklung ist allerdings nicht mit einem Anstieg der sogenannten klassischen psychiatrischen Krankheitsbilder, wie z. B. Psychosen, Schizophrenien oder endogene Depressionen, vereinbar, sondern vielmehr mit Gesundheitsstörungen im psychosomatischen Bereich, deren Ursachen vielfältiger Natur sind.

Die Zunahme der Renten ist bei jüngeren Versicherten besonders gross. Eine Analyse nach Altersklassen zeigt zudem, dass bis zur Gruppe der 40- bis 44jährigen mehr Renten wegen psychischer Krankheiten zugesprochen werden als wegen anderer Krankheiten (Abb. 2).

Vor allem bei jungen Erwachsenen, die noch ein langes Erwerbsleben vor sich hätten, wirkt sich das frühe Ausscheiden aus dem Erwerbsleben doppelt schlimm aus. Oftmals geht die definitive Ausgliederung aus dem Erwerbsleben für die Betroffenen auch mit einer Verschlechterung der Lebensqualität und mit sozialer Ausgrenzung einher. Auf der anderen Seite sind die Renten über einen viel längeren Zeitraum auszubezahlen und zu finanzieren als bei älteren Versicherten.

\section{Frühzeitiger handeln}

Eines der Hauptziele der 5. IV-Revision ist es, mehr Menschen mit gesundheitlichen Problemen - insbesondere im psychischen Bereich - im Erwerbsleben zu behalten oder wieder einzugliedern. Heute bezahlt die IV zum Teil Renten, obwohl die Versicherten mit der richtigen, vor allem aber rechtzeitigen Unterstützung zumindest teilerwerbstätig sein könnten. Eine wichtige Ursache für die ungenügende Eingliederung ist die Tatsache, dass nicht nur die meisten Versicherten, sondern auch die behandelnden Ärzte eher nicht wissen, dass die Invalidenversiche-

\section{$5^{\mathrm{e}}$ révision de l'Al: quel rôle jouent les médecins?}

Le nombre des rentiers $\mathrm{Al}$ a fortement augmenté ces dix dernières années; une personne sur vingt figure aujourd'hui au nombre des rentiers Al. Les rentes accordées pour des maladies psychiques ont très largement dépassé la moyenne.

L'un des objectifs principaux de la $5^{\mathrm{e}}$ révision de l'Al est de maintenir ou réintégrer de plus en plus de gens au travail avec leurs problèmes de santé, même psychiques.

Plus l'intégration professionnelle sera rapide, et plus les chances de succès seront grandes. Les nouveaux instruments préconisés pour assurer la rapidité de la saisie du cas et de l'intervention devraient y aider, de même que les mesures d'intégration.

Afin de pouvoir atteindre l'objectif fixé par la $5^{\mathrm{e}}$ révision de I'Al, une bonne collaboration entre les différents groupes de médecins impliqués sera indispensable. Le médecin traitant est un partenaire important des assurés, car il est le premier à décider sur la possibilité ou non de travailler de l'intéressé. Pour les services cantonaux de I'Al, il est un partenaire important dans ses efforts d'intégration. La responsabilité, sur le plan de l'assurance médicale, est portée par les services médicaux régionaux (SMR), qui occupent un rôle de médiateur entre la médecine, le droit et l'assurance.

rung (IV) versicherte Personen mit gesundheitlich bedingter Arbeitsunfähigkeit in der beruflichen Eingliederung und Wiedereingliederung unterstützen kann. So melden sich Betroffene in über 90\% der Fälle erst, nachdem sie bereits mehr als ein Jahr nicht mehr gearbeitet und oftmals bereits ihren Arbeitsplatz verloren haben. Die Chancen für eine erfolgreiche Wiederaufnahme einer Arbeit sinken aber mit jedem Tag Abwesenheit vom Arbeitsplatz und liegen nach einem Jahr bereits unter $20 \%$. 
Abbildung 1

Anteil der IV-Rentner/innen an der aktiven Bevölkerung (18 bis Rentenalter AHV).

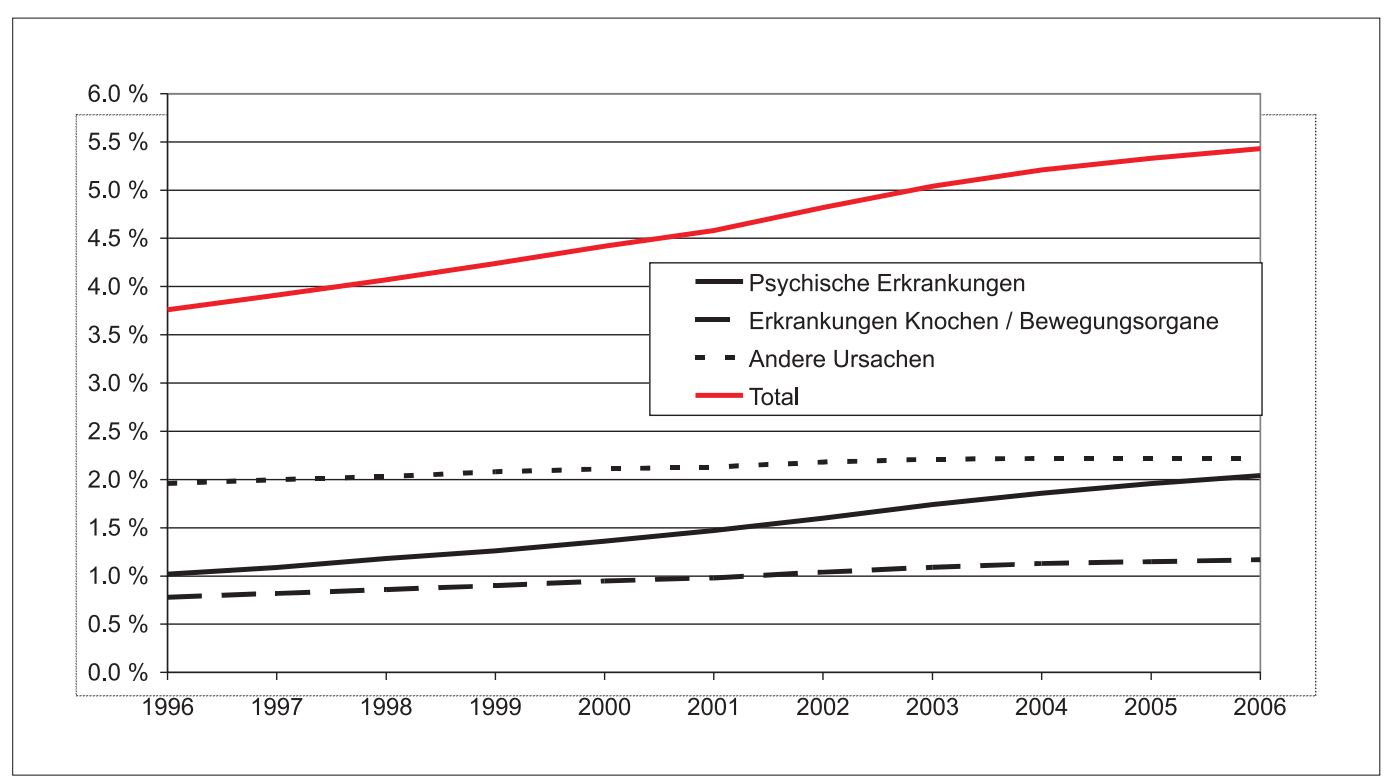

Abbildung 2

IV-Neurenten wegen Krankheiten in der Schweiz 2005.

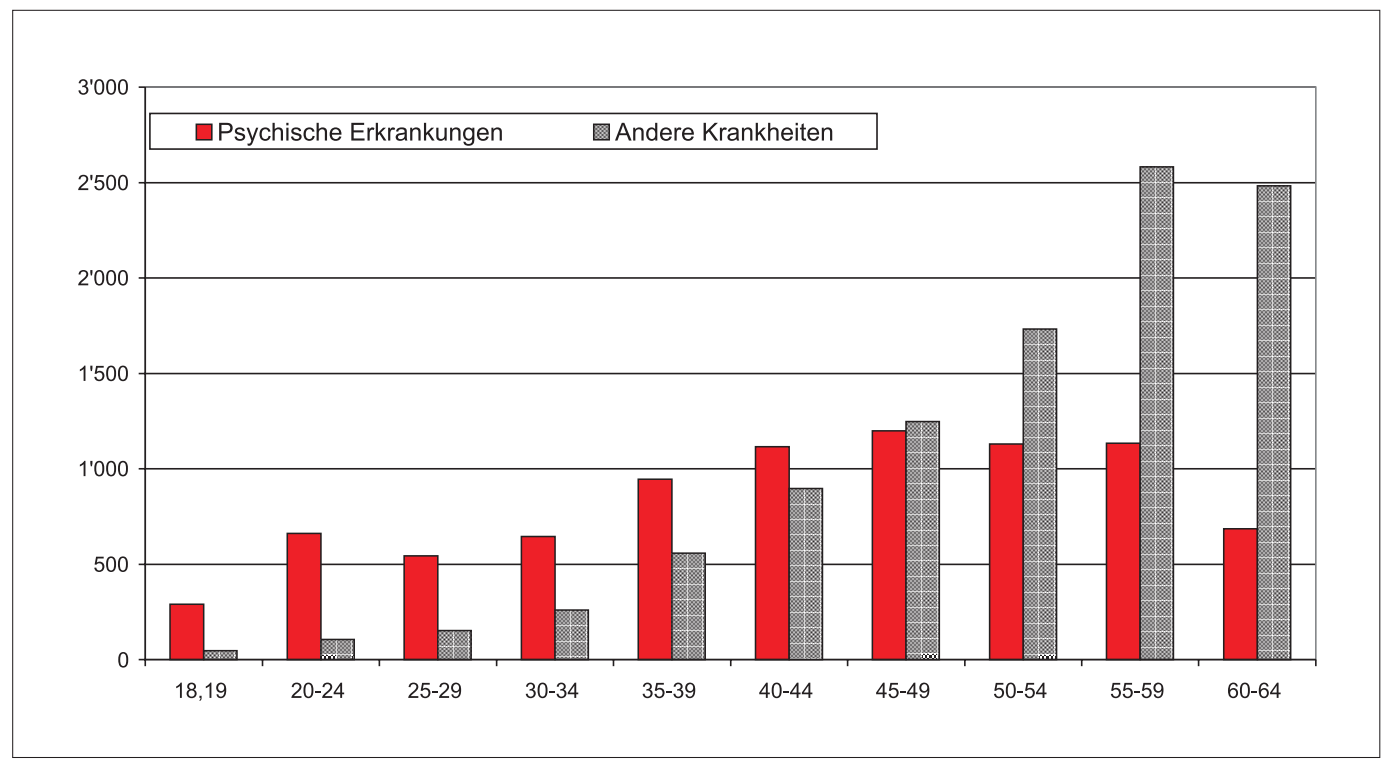

Unterstützung durch behandelnde Ärzte

Wenn Betroffene dank frühzeitiger und gezielter Unterstützung zumindest teilerwerbstätig bleiben, wird der Ausgrenzung mit ihren negativen Folgen vorgebeugt. Gerade auch um der sozialen Ausgrenzung entgegenzuwirken, ist der behandelnde Arzt ein guter Partner der Versicherten, weil er als erster richtungsweisend über Arbeiten oder Nichtarbeiten der Betroffenen entscheidet [2]. Die Beurteilung der gesundheitlichen Beeinträchtigung, die zur Arbeitsunfähigkeit führt, ist primär eine ärztliche Aufgabe. Allein aufgrund dieser Tatsache ist heute auch der behandelnde Arzt gefordert, sich neben der ärztlichen Behandlung auch mit den Konsequenzen ärztlicher Zeugnisse auseinanderzusetzen.

Eine längerdauernde Arbeitsabsenz hat nicht nur wirtschaftliche und soziale, sondern auch gesundheitliche Folgen. Beispielsweise führt eine schmerzbedingte Schonhaltung häufig bereits nach wenigen Wochen $\mathrm{zu}$ chronischen Beschwerden im unteren Rückenbereich. Nach längerer Dauer der Arbeitsunfähigkeit entwickelt sich nicht selten aus dem ursprünglich soma- 
tischen Rückenleiden zusätzlich eine depressive Störung und schliesslich eine schwere Depression, die oft mit einem vollständigen Verlust der Tagesstruktur einhergeht und in einer Erwerbsunfähigkeit münden kann [2]. Realistischerweise ist in diesen Situationen eine Reintegration in den beruflichen Alltag stark erschwert, wenn nicht sogar unmöglich; eine Rente ist dann die «Ultima ratio».

In solchen Situationen kann der praktizierende Arzt als Vertrauensperson der Versicherten nützliche Aufklärungsarbeit leisten, nicht nur betreffend die Auswirkungen gesundheitlicher Störungen auf die Situation am Arbeitsplatz, sondern auch betreffend die Möglichkeit, Leistungen bei der IV zu beziehen, bevor der Verlust des Arbeitsplatzes infolge Arbeitsunfähigkeit eintritt. Mit der 5. IV-Revision rückt die Eingliederung gegenüber der Rente noch deutlicher in den Vordergrund als bisher. Die beste Voraussetzung für eine erfolgreiche Eingliederung ist die möglichst frühe Erfassung und frühe berufliche Integration der in ihrer Arbeitsfähigkeit beeinträchtigten Versicherten, also in einem Stadium, in dem die Prognose noch günstig ist. Im Rahmen der medizinischen Behandlung und Betreuung hat der behandelnde Arzt eine gute Vertrauensbasis mit den Versicherten aufgebaut. Damit wird er auch zu einem wichtigen Partner für die kantonalen IV-Stellen im Rahmen ihrer Eingliederungsbemühungen. Kann er doch mit seiner auf Vertrauen basierenden Beziehung zu den Versicherten in der Früherfassung und Frühintegration bei gesundheitlich beeinträchtigten Personen an einer noch rechtzeitigen, angepassten beruflichen Integration aktiv mitwirken und damit auch einer allfälligen Chronifizierung des Leidens vorbeugen.

Zur Unterstützung von Versicherten in den beruflichen Einstieg oder Wiedereinstieg bietet die IV im Rahmen der 5. IV-Revision neue Integrationsmassnahmen an [1]. Zu diesen zählen unter anderem

- die sozialberuflichen Rehabilitationsmassnahmen, mit denen die Eingliederungsfähigkeit einer Person durch die Gewöhnung an den Arbeitsprozess, Aufbau der Arbeitsmotivation, Stabilisierung der Persönlichkeit und das Einüben sozialer Grundelemente verbessert wird. $\mathrm{Zu}$ den Modulen der sozialberuflichen Rehabilitation zählen das Belastbarkeits- und Aufbautraining sowie die wirtschaftsnahe Integration mit Support am Arbeitsplatz (WISA);

- Beschäftigungsmassnahmen: Wenn eine Person nach Erreichen der Eingliederungsfähigkeit auf den Beginn einer Umschulung warten muss, noch keine Arbeitsstelle gefunden hat oder Gefahr läuft, die Eingliederungsfähigkeit zu verlieren, werden Beschäftigungsmassnahmen zur Aufrechterhaltung einer Tagesstruktur durchgeführt. Das entsprechende Instrument ist die Arbeit zur Zeitüberbrückung.

\section{RAD-Ärzte informieren die regionale Ärzteschaft}

Eine wichtige Aufgabe der regionalen ärztlichen Dienste (RAD) besteht darin, die Kooperation mit den in der jeweiligen Region praktizierenden Ärzten gezielt zu fördern und sie über die neuen Leistungen der IV zu informieren.

Die Ärzte der RAD übernehmen als Vermittler zwischen Medizin, Recht und Versicherung die versicherungsmedizinische Verantwortung. Damit unterscheidet sich ihre Aufgabe grundsätzlich von derjenigen der behandelnden Ärzte. Während letztere für die medizinische Diagnostik und Behandlung zuständig sind, befasst sich der RAD-Arzt im wesentlichen mit der Erfassung des Gesundheitsschadens und dessen Auswirkungen auf die Arbeitsfähigkeit in der aktuellen oder in einer angepassten Tätigkeit. Diese Aufgabe setzt nicht nur eine mehrjährige klinische Tätigkeit voraus, sondern zusätzlich auch fundierte Kenntnisse der Versicherungsmedizin. Dieses Wissen holt sich der RAD-Arzt einerseits am Arbeitsplatz selbst, andererseits aber auch über die zunehmenden Angebote der Swiss Insurance Medicine [3] und der Academy for Swiss Insurance Medicine [4]. Gerade wegen seiner durch die 5. IV-Revision gesetzlich geregelten Aufgabe, bei den Versicherten die zumutbare Arbeitsfähigkeit zu beurteilen, steht der RADArzt heute in einer vermeintlichen Konkurrenzsituation mit den behandelnden Ärzten. Wird man sich bewusst, dass der RAD-Arzt und der behandelnde Arzt unterschiedliche Aufgaben haben, dann entspannt sich dieses Verhältnis. Der versicherungsmedizinische Ansatz schliesst aus versicherungsrechtlichen Gründen rein soziale Faktoren als Ursache einer Arbeitsunfähigkeit von den IV-Leistungen aus: Rein soziale oder wirtschaftliche Gründe führen zur Leistungspflicht der Arbeitslosenversicherung bzw. der Sozialhilfe, nicht jedoch der IV.

Mit ihren Aufgaben sind die RAD heute vielfältig gefordert. Sie liefern die für den Rentenentscheid relevanten versicherungsmedizinischen Grundlagen. Für die Beurteilung der stetig komplexer werdenden Gesundheitsschäden haben sie die erforderliche (und erst durch die 4. IV-Revision gesetzlich geregelte) Untersuchungskompetenz. Weiter kommen sie auch immer häufiger in der Phase der Früherfassung von Versicherten 
als Mitglied eines Teams mit anderen Spezialisten der IV zusammen zum Einsatz.

\section{Zusammenwirken aller Ärztegruppen erforderlich}

Damit das mit der 5. IV-Revision anvisierte Ziel, das bestehende Eingliederungspotential maximal auszuschöpfen, erreicht werden kann, ist ein gutes Zusammenwirken aller beteiligten Ärztegruppen unerlässlich. So greifen die Eingliederungsmassnahmen der IV nur dann, wenn Personen mit einer gesundheitsbedingten Arbeitsplatzabsenz frühzeitig erfasst und die Eingliederungsbemühungen der IV frühzeitig erfolgen können. Dies setzt unter anderem eine hohe Kooperation zwischen den Versicherungsmedizinern und den behandelnden Ärzten voraus. Zunehmend ist aber für eine optimale Betreuung und Begleitung des Versicherten auch die interdisziplinäre Zusammenarbeit zwischen niedergelassenen Ärzten, den Ärzten der RAD sowie anderen Fachspezialisten der kantonalen IV-Stellen für eine erfolgreiche Eingliederung oder Wiedereingliederung im Rahmen von sogenannten Case Teams unerlässlich. Durch ihre aktive Mitwirkung in der Früherfassung und Frühintegration versicherter Personen ermöglichen die RAD-Ärzte zusammen mit den niedergelassenen Ärzten den Betroffenen den Weg zurück in den Arbeitsprozess und das Aufrechterhalten einer adäquaten Lebensqualität.

\section{Literatur}

1 Bundesamt für Sozialversicherungen. Dokumentation zur 5. IV-Revision vom 17. März 2007. www.bsv.admin.ch.

2 Bohny CL. Früherfassung von Arbeitsfähigkeit: Was bringt die 5. IV-Revision? Schweiz Ärztezeitung. 2004;85(38):2031-3.

3 Swiss Insurance Medicine. www.swiss-insurancemedicine.ch.

4 Academy of Swiss Insurance Medicine. www.asim. unibas.ch. 